\title{
Dysconnectivity of a brain functional network was associated with blood inflammatory markers in depression
}

\author{
Athina R. Aruldass ${ }^{\mathrm{a}, *}$, Manfred G. Kitzbichler ${ }^{\mathrm{a}}$, Sarah E. Morgan ${ }^{\mathrm{a}}$, Sol Lim ${ }^{\mathrm{a}}$, Mary-Ellen Lynall ${ }^{\mathrm{b}}$, \\ Lorinda Turner $^{\mathrm{b}}$, Petra Vertes ${ }^{\mathrm{a}}$, Wellcome Trust Consortium for Neuroimmunology of Mood \\ Disorders and Alzheimer's Disease (NIMA), Jonathan Cavanagh ${ }^{\mathrm{c}}$, Phil Cowen ${ }^{\mathrm{d}}$, \\ Carmine M. Pariante $^{\mathrm{e}}$, Neil A. Harrison ${ }^{\mathrm{f}}$, Edward T. Bullmore ${ }^{\mathrm{a}}$

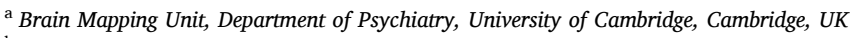 \\ ${ }^{\mathrm{b}}$ Department of Medicine, University of Cambridge, UK

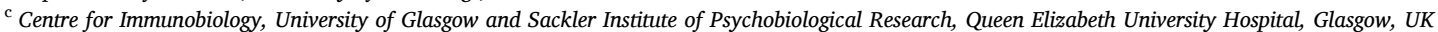 \\ ${ }^{\mathrm{d}}$ University of Oxford Department of Psychiatry, Warneford Hospital, Oxford, UK \\ ${ }^{\mathrm{e}}$ Stress, Psychiatry and Immunology Laboratory \& Perinatal Psychiatry, Maurice Wohl Clinical Neuroscience Institute, Kings College London, UK \\ ${ }^{\mathrm{f}}$ Cardiff University Brain Research Imaging Centre, Cardiff University, Maindy Road, Cardiff, UK
}

\section{A R T I C L E I N F O}

\section{Keywords:}

Functional connectivity

Network-based statistics

Inflammation

Depression

\begin{abstract}
A B S T R A C T
Objective: There is increasing evidence for a subgroup of major depressive disorder (MDD) associated with heightened peripheral blood inflammatory markers. In this study, we aimed to understand the mechanistic brainimmune axis in inflammation-linked depression by investigating associations between functional connectivity (FC) of brain networks and peripheral blood immune markers in depression.

Methods: Resting-state functional magnetic resonance imaging (fMRI) and peripheral blood inflammatory markers (C-reactive protein; CRP, interleukin-6; IL-6 and immune cells) were collected on $\mathrm{N}=46$ healthy controls (HC; $\mathrm{CRP} \leq 3 \mathrm{mg} / \mathrm{L}$ ) and $\mathrm{N}=83$ cases of depression, stratified further into low CRP cases (loCRP cases; $\leq 3 \mathrm{mg} / \mathrm{L} ; \mathrm{N}=50$ ) and high CRP cases (hiCRP cases; $>3 \mathrm{mg} / \mathrm{L} ; \mathrm{N}=33$ ). In a two-part analysis, network-based statistics (NBS) was firstly used to ascertain whole-brain FC differences in HC vs hiCRP cases. Secondly, we investigated the association between this network of interconnected brain regions and continuous measures of peripheral CRP $(\mathrm{N}=83)$, IL-6 $(\mathrm{N}=72)$, neutrophils and $\mathrm{CD}^{+}{ }^{+}$-cells $(\mathrm{N}=36)$ in depression cases only. Results: Case-control NBS testing revealed a single network of abnormally attenuated FC in the high CRP depression cases compared to healthy controls. Connections within this network were mainly between brain regions located in the left insula/frontal operculum and posterior cingulate cortex, which were assigned to ventral attention and default mode canonical fMRI networks respectively. Within-group analysis across all depression cases, secondarily demonstrated that FC within the identified network significantly negatively scaled with CRP, IL-6 and neutrophils.

Conclusions: The findings suggest that inflammation is associated with disruption of functional connectivity within a brain network deemed critical for interoceptive signalling, e.g. accurate communication of peripheral bodily signals such as immune states to the brain, with implications for the pathogenesis of inflammation-linked depression.
\end{abstract}

\section{Introduction}

Subsuming a heterogeneous population of patients into diagnostic categories that are chiefly defined by syndromic and behavioural constructs, as opposed to biological discriminators, has been a persistent issue in classification of major depressive disorder (MDD; henceforth also referred to as depression) (Schmaal et al., 2020). In this context, there has been growing interest in identifying a subgroup of MDD cases associated with blood biomarkers of peripheral inflammation (KiecoltGlaser et al., 2015; Otte et al., 2016), so-called inflammation-linked

\footnotetext{
* Corresponding author.

E-mail address: ara49@cam.ac.uk (A.R. Aruldass).
} 
depression.

Evidence for mechanistic links between the immune system and depression was first reported about 30 years ago, and has since become the foundation of the emerging field of immunopsychiatry (Maes et al., 1992; Dantzer et al., 2008; Miller and Raison, 2016; Khandaker et al., 2017; Pariante, 2015; Khandaker et al., 2021). Meta-analyses of crosssectional, case-control studies have demonstrated low-grade increases in peripheral C-reactive protein (CRP), inflammatory cytokines, particularly interleukin 6 (IL-6) and innate immune cells in MDD cases (Haapakoski et al., 2015; Osimo et al., 2020; Lynall et al., 2020). In longitudinal studies, higher levels of CRP and IL- 6 at baseline predicted increased risk of depression at follow-up, suggesting a causal role for inflammation in depression (Khandaker et al., 2014). While there is a clear implication for inflammation in the pathoetiology of a subgroup of MDD cases, but what this mechanistically entails across the neuroimmune axis still remains elusive (Nusslock and Miller, 2016; Wohleb et al., 2016). Functional magnetic resonance imaging (fMRI) is a potentially useful tool for investigation of inflammation- and depression-related changes in brain functional connectivity (Logothetis, 2008; Fox and Greicius, 2010).

In case-control fMRI studies of depression, functional connectivity (FC) abnormalities have been frequently reported with a focus on canonical resting state networks (RSNs) such as the default mode network (DMN), the ventral attentional network (VA), and the fronto-parietal control network (FP), each of which is associated with specific behavioral domains and/or classes of symptoms relevant to MDD (Thomas Yeo et al., 2011). For example, abnormal connectivity within the DMN has been linked to rumination and negative self-referential thoughts (Sheline et al., 2009; Hamilton et al., 2015); whereas dysconnectivity of the VA network has been associated with impairments in emotion recognition and processing, apathy and anhedonia (Seeley, 2019). Nonetheless, there are inconsistencies between individual studies. Both abnormal hypoconnectivity (reduced positive FC and increased negative FC), and abnormal hyperconnectivity (increased positive FC and reduced negative FC), have been reported for the DMN, FP and VA networks in depression (Kaiser et al., 2015).

There have been fewer fMRI studies of inflammation-related changes in resting state connectivity, with greater paucity in depression cases. However, a recent meta-analysis encompassing human experimental models of inflammation, clinical studies of hepatitis $\mathrm{C}$ patients receiving IFN $\alpha$ treatment, and observational studies of community samples with variable blood levels of CRP, reported that inflammation-related changes were consistently co-localized to DMN, VA and limbic functional networks (Kraynak et al., 2018). Seminal studies have reported CRP-related differences in connectivity among depression cases (rather than between cases and controls), where high CRP correlated negatively with seed-based analysis of cortico-striatal and cortico-amygdalar connectivity (Felger et al., 2016; Mehta et al., 2018; Yin et al., 2019). Overall, there is emerging evidence that peripheral inflammation can perturb FC of brain networks that are known to be critical for emotional regulation (Damasio et al., 2000; Critchley and Garfinkel, 2017; Savitz and Harrison, 2018).

Here, we investigated the relationships between depression, peripheral inflammation and whole-brain functional connectivity in two related analyses, complementary to our previous investigation on microstructural MRI and FC differences in HC and all depression cases, in the same imaging sample (Kitzbichler et al., 2020). First, we used network-based statistics (NBS) on whole-brain functional connectivity matrices or connectomes to test for network-level FC differences between high CRP depression cases (hiCRP cases; CRP > $3 \mathrm{mg} / \mathrm{L}$ ) compared to healthy controls (HC; CRP $\leq 3 \mathrm{mg} / \mathrm{L}$ ). Informed by prior reports of decreased FC associated with inflammation in depression (Felger et al., 2016; Mehta et al., 2018; Yin et al., 2019), and more generally with depression (Kaiser et al., 2015; Veer et al., 2010), we tested the one-tailed null hypothesis that there is no set of interconnected edges (or connections) with attenuated FC in hiCRP depression cases compared to HC. This null hypothesis was refuted. A network of significantly attenuated connections linking mainly insular, cingulate and subcortical regions was identified. Second, we used this network as a "mask" to explore within-group relationships between FC measured in all depression cases, and peripheral inflammation indexed by CRP, IL- 6 and two classes of immune cells (neutrophils and CD4 ${ }^{+} \mathrm{T}$ cells) which were previously shown to be significantly increased in casecontrol analysis of a larger sample from the same study (Lynall et al., 2020). We hypothesized that increased blood protein and cellular inflammatory markers would be negatively correlated with FC of this network.

\section{Methods and materials}

\subsection{Participants}

Biomarkers for Depression (BioDep) was an observational casecontrol study conducted as part of the Wellcome Trust Neuroimmunology of Mood Disorders and Alzheimer's disease (NIMA) Consortium. All procedures were approved by an independent national research ethics service (NRES) committee (NRES: East of England, Cambridge Central, UK; Reference: 15/EE/0092) and all participants provided written informed consent.

All participants satisfied inclusion criteria, e.g. aged 25-50 years, and exclusion criteria, e.g. major medical inflammatory disorder or immuno-modulatory medication (Supplementary Appendix; SA1). The adult age range was motivated by the intention of this study to discover brain imaging biomarkers that could be useful immediately for clinical trials of novel anti-inflammatory drugs for depression. Noting that such trials typically focus on the adult population (rather than adolescent or older demographics), we pragmatically specified the age range for this study to align with the likely demographic profile of participants in future clinical trials with fMRI-based surrogate endpoints. All depression cases screened positive for current depressive symptoms on the Structured Clinical Interview for DSM-5 Depressive Disorders (SCID), and had total Hamilton Rating Scale for Depression (HAMD-17) score > 13 (SA13).

After initial telephone screening, potentially eligible participants attended an eligibility assessment (Fig. S1), including blood sampling for CRP assay, at one of 5 UK recruitment centers (Brighton, Cambridge, Glasgow, King's College London (KCL), or Oxford). Eligible participants next attended one of 3 UK assessment centers (Cambridge, KCL or Oxford) for venous blood sampling, clinical assessment, and MRI scanning, all scheduled on the same day at 8-10am (SA2). Depression cases were then stratified by blood CRP level: loCRP depression cases had CRP $\leq 3$ $\mathrm{mg} / \mathrm{L}$ (corresponding to "low" and "average" risk for cardiovascular disease per American Heart Association (AHA) guidelines), hiCRP depression cases had CRP $>3 \mathrm{mg} / \mathrm{L}$ ("high" risk for cardiovascular disease per AHA) (Pearson et al., 2003). We note that the high/low CRP cutoff value of $3 \mathrm{mg} / \mathrm{L}$ has been widely used in other studies of inflammation-related depression (Wium-Andersen et al., 2013; Raison et al., 2013; Uher et al., 2014). Three hiCRP depression cases with CRP $>10 \mathrm{mg} / \mathrm{L}(10.2-11.4 \mathrm{mg} / \mathrm{L})$ were retained for analysis as there was no clinical evidence for infection or other exclusionary medical disorders (SA1). All HCs had CRP $\leq 3 \mathrm{mg} / \mathrm{L}$.

\subsection{Blood immune biomarkers}

All participants provided up to $90 \mathrm{~mL}$ of fasting venous blood. CRP was measured using high sensitivity immunoturbidometry at a central laboratory $\left(Q^{2}\right.$ Solutions, Livingston, Scotland, UK). Cytokine and chemokine levels were measured in plasma and serum using relevant VPLEX 10-spot immunoassay kits from Meso Scale Discovery (MSD; Rockville, MD , USA) (see Section S2). After quality control (QC), analyzable cytokine data on IL- 6 and other cytokines were obtained for $\mathrm{N}=72$ MDD cases (Table S1A). Only IL- 6 was available for analysis in 
the present study. All blood protein concentrations were logtransformed (base 10) prior to further analyses.

\subsection{Cellular biomarkers}

Absolute cell counts were available for 12 leukocyte classes for $\mathrm{N}=$ 36 depression cases (Table S1B-C) that were a subset of a previous report on a larger sample (Lynall et al., 2020). Absolute hematology cell counts (red cells, platelets, neutrophils, eosinophils, basophils, lymphocytes, monocytes) were measured centrally by a clinical diagnostics laboratory $\left(Q^{2}\right.$ Solutions, Livingston, Scotland, UK) for all participants across the 5 clinical centers. Flow cytometry was performed on fresh peripheral blood mononuclear cells (PBMCs) using live-dead stain and antibodies against CD3, CD4, CD8, CD19, CD56, CD14, and CD16 (see Supplementary Data Section S2) to estimate proportional or percentage counts for CD4 ${ }^{+}$T-cells, CD8 ${ }^{+}$T-cells, B-cells, CD16 $6^{\text {hi/bright }} \mathrm{NK}$ cells, CD56 $6^{\text {hi }}$ bright NK cells, NK T-cells, intermediate monocytes and classical monocytes. The immunophenotyping protocol e.g. sample preparation, staining and gating strategy for flow cytometry is detailed in (Lynall et al., 2020). Absolute counts for each sub-class of cells were derived by multiplying proportional count of each of these sub-classes of lymphocytes or monocytes (from flow cytometry) with the absolute count of the appropriate cell class (from hematology panel). For example, the proportional counts of monocyte sub-classes (from cytometry) were multiplied by the absolute count of (all) monocytes to estimate the absolute counts of intermediate and classical monocyte subclasses from hematology panel. Absolute cell counts were used rather than relative cell counts (as estimated directly by cytometry) because relative counts (reported in percentages or estimated as ratios of an index cell class in proportion to other cell classes) are sensitive to variation in both other cell classes and denominator (total white blood cell population). Thus, changes in relative counts i.e. proportional increase or decrease, are more ambiguously interpretable than absolute cell counts. We recognized a priori that some classes of immune cells have already been implicated in case-control studies of inflammation-related depression. Hence, we focused initially on two immune cell classes - neutrophils and $\mathrm{CD}^{+}$(helper) T-cells - since these are principal effectors of innate and adaptive immune responses, respectively (Mantovani et al., 2011; Glimcher and Murphy, 2000), and were both significantly increased in depression cases as reported in a prior case-control analysis of a larger sample drawn from the same study (Lynall et al., 2020).

We also used binary classification outcomes defined a priori from the preceding study (Lynall et al., 2020). Multivariate Gaussian mixture modelling and consensus clustering was performed on all absolute cellcounts of depression classes to define two subgroups with different immune cell profiles (see Supplementary Data Section S2 for further details on method). This fitting resulted in assignment of each depression case into "high-cell count" or "low-cell count" depression representative clusters (Fig. S3). We then used this designation to explore betweengroup difference in functional connectivity in the current smaller sample (see 2.7 Part 2 Methods below). The high-cell count depression subgroup had significantly higher concentrations of myeloid cells (neutrophils, basophils, eosinophils, and classical monocytes) and NK Tcells, and nominally increased counts of lymphoid cells. The high cell count group also had significantly increased CRP and nominally increased IL- 6 concentrations, compared to the low-cell count depression subgroup. Detailed data on absolute cell counts for the low-cell count subgroup $(\mathrm{N}=20)$ and the high-cell count subgroup $(\mathrm{N}=16)$ are reported in Table S1C.

\subsection{Functional magnetic resonance imaging}

Resting-state fMRI data were acquired using multi-echo (ME) echoplanar imaging (EPI) sequence with: relaxation time $(T R)=2.57 \mathrm{~s}$; echo times $\left(\mathrm{TE}_{1,2,3}\right)=15 \mathrm{~ms}, 34 \mathrm{~ms}$ and $54 \mathrm{~ms}$; acquisition time $=10 \mathrm{mins}$ $42.5 \mathrm{~s}=250$ time points in each fMRI time series. ME-EPI data were collected as 32 slices at -30 degrees to the AC-PC line with field of view $=240 \mathrm{~mm}$ and matrix size $=64 \times 64$, for voxel resolution of $3.75 \times 3.75$ $\times 3.99 \mathrm{~mm}$. The first six volumes were discarded and remaining data preprocessed using multi-echo independent component analysis (MEICA; (Kundu et al., 2012; Kundu et al., 2013) in AFNI. Images were then regionally parcellated using a 180 bilateral cortical surface-based atlas (Glasser et al., 2016) and 8 bilateral non-cortical regions from FreeSurfer (Fischl et al., 2002; Fischl, 2012), giving 376 regions in total. Timeseries were then bandpass filtered at wavelet scales 2 and 3 corresponding to $0.02-0.1 \mathrm{~Hz}$. The resulting wavelet coefficients were correlated pairwise between regions using Pearson's correlation coefficient to obtain a $376 \times 376$ symmetric FC matrix for each subject. FC matrices were then Fisher r-to-z transformed. Subjects with high degree of head motion estimated by framewise displacement, $\mathrm{FD}_{\max }>1.3 \mathrm{~mm}$ and/or $\mathrm{FD}_{\mathrm{rms}}>0.3 \mathrm{~mm}$, were excluded $(\mathrm{N}=4)$. Additional nuisance variables i.e. $\mathrm{FD}_{\mathrm{rms}}$, scan site and age were regressed edge-wise from the FC matrices (Fig. S4A).

\subsection{Part 1 analyses: Network-based statistics (NBS) and between-group difference in network connectivity}

NBS was implemented using the NBS MATLAB Toolbox (Zalesky et al., 2010) (Section 4 Supplementary Data). We first performed NBS case-control comparison on HC vs hiCRP depression cases. We used onetailed t-tests (HC > hiCRP depression cases) and performed 5000 random permutations. One-tailed hypothesis testing was justified by prior reports of decreased FC associated with inflammation in depression (Felger et al., 2016; Mehta et al., 2018; Yin et al., 2019), and more generally with depression (Kaiser et al., 2015; Veer et al., 2010). The test statistic threshold was initially set to $t_{\text {primary }}=3.0$, corresponding to nominal uncorrected $P=0.005$, and was reported to yield consistent findings across parcellation schemes (Zalesky et al., 2012; Cocchi et al., 2014). It was then increased by 0.1 step-wise, to retain edges with the strongest case-control differences i.e. greatest t-statistics, constituting a statistically significant subset of inter-connected nodes. NBS results at $\mathrm{t}_{\text {primary }}=3.8$ are reported here and presented as three-dimensional network visualizations using BrainNet Viewer (Xia et al., 2013). This case-control difference network was subsequently used as a "mask" for Part 2 analyses of the relationships between peripheral immune markers and functional connectivity within the group of all depression cases.

\subsection{Modular functional and areal anatomical decomposition of NBS network}

To gain more resolution on composition of resultant NBS network, predefined Yeo modular (Thomas Yeo et al., 2011) and Glasser anatomical areal assignments (Glasser et al., 2016) were used for functional and anatomical decomposition, respectively, of constituent brain regions (or nodes) within the NBS network. The fine-grained 180 bilateral cortical parcels (brain regions or nodes) from the Glasser et al. (Glasser et al., 2016) (Glasser et al., 2016) atlas were also described according to the more coarse-grained 22 anatomical areas reported in the original publication (see Supplementary Data Section S3B). Additionally, each parcel (brain region or node) was also assigned to one of the 7 functional modules i.e. visual (V), somatomotor (SM), dorsal attention (DA), ventral attention (VA), limbic (L), frontoparietal control (FP), and default mode network (DMN), as described in Yeo et al. (2011), based on its co-localisation with these canonical resting-state networks. The remaining 16 subcortical regions defined by FreeSurfer were assigned to a subcortical (SC) module. Details of both anatomical and functional node-labelling schemes are in Supplementary Data Section S3B (Fig. S4C-D). 
2.7. Part 2 analyses: Association within depression cases between functional connectivity and blood immune biomarkers

In the second part of our investigation, we performed continuous analyses to examine relationship between functional connectivity and four peripheral immune biomarkers (CRP, IL-6, neutrophils and CD4 ${ }^{+} \mathrm{T}$ cells) in all depression cases. While the Part 1 analyses were based on data from healthy controls and a subset (high CRP) of depression cases, the Part 2 analyses were based on data from all depression cases (without controls). Hence, the Part 1 and Part 2 samples are partially overlapping but not identical, which mitigates the risk of circularity. We examined relationship between the two variables firstly at edge-level by estimating Pearson's correlation between functional connectivity of each edge within the case-control "mask" defined in Part 1 and each immune biomarker. Next, we examined association at network-level by estimating average network connectivity (mean across all connections) from the "mask" for each depression participant, and then performed two-tailed linear regression against each immune biomarker. Additionally, for the cellular portion of Part 2 analyses, we also used the casecontrol NBS "mask" for initial categorical FC comparison between the "high-cell count" and "low-cell count" depression subgroups.

\subsection{Statistical methods}

Functional connectivity estimated by inter-regional time series correlations were normalised by Fisher's r-to-Z transformation prior to all analyses. Effect sizes were also reported using Cohen's d. Case-control and within-group comparisons of FC distributions were estimated using two-sample Kolmogorov-Smirnov tests. For Part 1 sensitivity analyses, BMI, sex and tobacco consumption (smoking status) were individually controlled for by (i) regressing each covariate separately on the functional connectivity matrices, and (ii) subsequently repeating casecontrol NBS testing on the residualized matrices. Sensitivity analyses for Part 2 were performed using hierarchical linear regression to control for the additive effects of covariates including sex, BMI and smoking status in analysis of the relationship between FC and immune biomarkers within the group of all depression cases (Table S3B-D). More details on statistical methods e.g. collinearity diagnostics, and sensitivity analyses are provided in Supplementary Data (Section S7 Table S3A-D; Fig. S6; Fig. S8).

\section{Results}

\subsection{Sample characteristics}

After QC procedures, analysable fMRI and CRP data were available for $\mathrm{N}=129$ participants, comprising $\mathrm{N}=46 \mathrm{HC}, \mathrm{N}=50$ loCRP depression cases and $\mathrm{N}=33$ hiCRP depression cases. Sociodemographic and clinical variables are summarised in Table 1 (see SA5 for details). High CRP depression cases included proportionally more females and had higher BMI and more severe depression scores than loCRP depression cases. BMI was also significantly correlated with CRP $(r=0.57$, $\left.P_{\text {FDR }}=0.001\right)$ and IL-6 $\left(r=0.45, P_{\text {FDR }}<0.05\right)$. Therefore, sex and BMI were included as covariates in NBS testing and subsequent statistical modelling (Table S1A-C). Depression cases were not excluded if currently prescribed antidepressants and/or pre-specified concomitant medication for minor diagnostic comorbidities (see SA4 for details). Potential confounding effects of antidepressant and other medication and clinical comorbidities were controlled for by sensitivity analyses (Table S3B-C).

Group differences were estimated using Mann-Whitney $U$ test or chi-squared test. ${ }^{a}$ body mass index, $1 \mathrm{HC}$ missing data omitted in casecontrol statistical comparison; ${ }^{b}$ Hamilton Rating Scale for Depression; ${ }^{c}$ Beck's Depression Inventory (version II); ${ }^{d}$ Snaith-Hamilton Pleasure

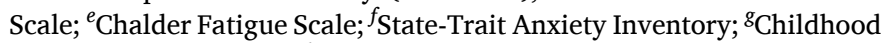
Trauma Questionnaire; ${ }^{h}$ Perceived Stress Scale; ${ }^{i}$ Life Events Questionnaire; ${ }^{j}$ high-sensitivity C-reactive protein. Healthy controls (CRP $\leq 3$ $\mathrm{mg} / \mathrm{L}$ ); all depression; all depression cases (CRP 3 - $10 \mathrm{mg} / \mathrm{L}$ ); loCRP depression; low CRP depression cases with CRP $\leq 3 \mathrm{mg} / \mathrm{L}$; hiCRP depression; high CRP depression cases with CRP > $3 \mathrm{mg} / \mathrm{L}$; IQR; interquartile range (Q3-Q1); '`statistical comparison performed using unpaired $t$-test; $* P<0.05 ;{ }^{* *} P<0.01 ;{ }^{* * *} P<0.001$.

Table 1

Sociodemographic, clinical and serological variables in the analyzable cohort $(\mathrm{N}=129)$.

\begin{tabular}{|c|c|c|c|c|c|c|c|c|c|c|}
\hline & \multicolumn{2}{|c|}{$\begin{array}{l}\text { Healthy controls } \\
\qquad(N=46)\end{array}$} & \multicolumn{2}{|c|}{$\begin{array}{l}\text { all depression } \\
\qquad(\mathrm{N}=83)\end{array}$} & \multirow{2}{*}{$\begin{array}{c}\begin{array}{c}\text { Case-Control } \\
\text { difference }\end{array} \\
p \text {-value }\end{array}$} & \multicolumn{2}{|c|}{$\begin{array}{l}\text { loCRP depression } \\
\qquad(\mathrm{N}=50)\end{array}$} & \multicolumn{2}{|c|}{$\begin{array}{l}\text { hiCRP depression } \\
\qquad(\mathrm{N}=33)\end{array}$} & \multirow{2}{*}{$\begin{array}{c}\begin{array}{c}\text { loCRP-hiCRP } \\
\text { difference }\end{array} \\
p \text {-value }\end{array}$} \\
\hline & Median & IQR & Median & IQR & & Median & IQR & Median & IQR & \\
\hline \multicolumn{11}{|l|}{ Sociodemographic / Clinical } \\
\hline Age (years) & 34.5 & 11.9 & 36.3 & 12.6 & 0.21 & 37.6 & 11.4 & 36.3 & 12.6 & 0.54 \\
\hline $\operatorname{BMI}\left(\mathrm{kg} / \mathrm{m}^{2}\right)^{a}$ & 23.5 & 5.3 & 26.6 & 5.9 & $0.001 * *$ & 25.4 & 4.6 & 27.8 & 7.4 & $0.001 * *$ \\
\hline Sex, Male $(n, \%)$ & 19 & 41.3 & 26 & 30 & 0.34 & 21 & 43.8 & 5 & 16.7 & $0.02 *$ \\
\hline Tobacco, current smokers $(n, \%)$ & 2 & 4.3 & 15 & 19.2 & $0.05^{*}$ & 11 & 22.9 & 4 & 13.3 & 0.39 \\
\hline Alcohol, current users $(n, \%)$ & 23 & 50 & 47 & 60.3 & 0.59 & 30 & 60 & 17 & 51.5 & 0.59 \\
\hline Cannabis, current users $(n, \%)$ & 4 & 8.7 & 5 & 6.4 & 0.95 & 4 & 8 & 1 & 3 & 0.65 \\
\hline Handedness, Right (n, \%) & 42 & 91.3 & 69 & 88.5 & 0.29 & 41 & 82 & 28 & 84.8 & 0.94 \\
\hline Ethnicity, White $(n, \%)$ & 31 & 67.4 & 67 & 85.9 & 0.55 & 43 & 86 & 24 & 72.7 & 0.24 \\
\hline \multicolumn{11}{|l|}{ Behavioral Instruments } \\
\hline HAM-D (17-items) $)^{b}$ & 0 & 1 & 17 & 7 & $<0.001 * * *$ & 17 & 7 & 18 & 5 & 0.44 \\
\hline $\mathrm{BDI}-\mathrm{II}^{\mathrm{C}}$ & 1 & 3 & 25 & 11.5 & $<0.001 * * *$ & 25 & 10.8 & 26 & 10 & 0.56 \\
\hline SHAPS $^{d}$ & 0 & 0 & 4 & 5.5 & $<0.001 * * *$ & 5 & 4 & 3 & 6 & 0.22 \\
\hline $\mathrm{CFS}^{e}$ & 11 & 1 & 20 & 7 & $<0.001^{* * *}$ & 20.5 & 7 & 20 & 6 & 0.94 \\
\hline STAI - S (items $1-20)^{f}$ & 25 & 8.8 & 51 & 15 & $<0.001 * * *$ & 52.5 & 15.5 & 49 & 12 & 0.63 \\
\hline STAI - T (items $21-40)^{f}$ & 28.5 & 9 & 61 & 11.5 & $<0.001^{* * *}$ & 61 & 11.8 & 61 & 12 & 0.95 \\
\hline $\mathrm{CTQ}^{g}$ & 37 & 5.8 & 51 & 22 & $<0.001 * * *$ & 53 & 20.8 & 47 & 16 & 0.06 \\
\hline $\mathrm{PSS}^{h}$ & 11.5 & 8 & 26 & 6 & $<0.001 * * *$ & 26.5 & 5.6 & 26 & 6 & 0.73 \\
\hline LEQ $(\text { score })^{i}$ & 0 & 1 & 1 & 1 & $<0.001 * * *$ & 1 & 2 & 1 & 1 & 0.61 \\
\hline LEQ (rating) ${ }^{i}$ & 1.5 & 1 & 3 & 4 & $<0.001^{* * *}$ & 3 & 4 & 3 & 3.5 & 0.91 \\
\hline \multicolumn{11}{|l|}{ Inflammatory Biomarkers } \\
\hline $\mathrm{CRP}(\mathrm{mg} / \mathrm{L})^{j}$ & 0.6 & 1.1 & 1.8 & 3.5 & $<0.001 * * *$ & 0.8 & 1.0 & 4.5 & 3.7 & $<0.001 * * *$ \\
\hline CRP $\left(\log _{10} \mathrm{mg} / \mathrm{L}\right)^{j \dagger}$ & -0.2 & $(0.4)$ & 0.2 & $(0.5)$ & $<0.001 * * *$ & -0.1 & $(0.4)$ & 0.7 & $(0.2)$ & $<0.001 * * *$ \\
\hline
\end{tabular}




\subsection{Case-control network-level differences in FC (Part 1)}

Case-control comparison between hiCRP depression cases and HC, using NBS yielded a single network comprising 38 edges (or connections) and 33 nodes (or regions) (one-tailed $\mathrm{P}=0.043$, Cohen's $\mathrm{d}=$ 0.45) (Fig. 1A). Functional connectivity over all edges in the network was less negative in HC $(-0.22<\mathrm{Z}<0.51)$, than in loCRP depression cases $(-0.35<\mathrm{Z}<0.46)$ and hiCRP depression cases $(-0.46<\mathrm{Z}<0.31)$ (Fig. 1B-C). Distribution of edge-weights was significantly different between hiCRP depression cases vs HC (KS test, $P=1.6 \times 10^{-12}, \mathrm{D}=0.61$, Cohen's d $=1.13$ ), loCRP depression cases vs HC (KS test; $P=0.00027$, $\mathrm{D}=0.34$, Cohen's $\mathrm{d}=0.47$ ) and hiCRP depression vs loCRP depression (KS test; $P=0.00027, \mathrm{D}=0.34$, Cohen's $\mathrm{d}=0.61$ ).

The nodes of this network were mainly located within the insular/ frontal opercular and posterior cingulate cortical areas based on the Glasser et al. (2016) anatomical area assignment, each assigned to the VA and DMN module respectively, based on the Yeo-7 functional network assignment (each network referred to as a "module") (Thomas Yeo et al., 2011). Edges between DMN and VA nodes (10/38) were the most abundant between-module connections, followed by DMNSomatomotor (8/38) edges (Table S2A-B, Fig. S5A). Topological representation of the network further confirmed that edges with attenuated FC were mainly coupling insular/frontal opercular nodes of the VA module, e.g., L_FOP4, and posterior cingulate cortical nodes of the DMN module, e.g., L_d23ab and L_31 pv (Fig. 1D; Table S2A).

To contextualize this network in light of prior literature, we queried the BrainMap database (Fox and Lancaster, 2002) to identify fMRI studies that showed co-activation for both insula and cingulate cortices. The 8 resultant studies (Table S2C) involved subjective perception of various stimuli, self-referential and salience processing, all of which are interoceptive processes (Damasio and Carvalho, 2013). When casecontrol analysis by NBS was repeated after controlling for potentially confounding covariates by prior regression on the functional connectivity matrices, there were no significant differences identified after controlling for BMI or sex. But the pattern of case-control difference was substantially unchanged after controlling for smoking status (Fig. S5B).

\subsection{Association between FC and inflammatory proteins in depression (Part 2)}

We next examined the relationships between inflammatory proteins and edge-wise FC within the mask defined by the case-control analysis (HC vs hiCRP depression cases NBS testing), for all depression cases. Both IL-6 $(-0.49<\mathrm{r}<0)$ and CRP $(-0.48<\mathrm{r}<0)$ were negatively correlated with functional connectivity of edges within the network (Fig. 2A, first column). When testing each edge separately for association with each protein, while controlling for multiple comparisons with FDR $=5 \%, 21$ edges were significantly negatively correlated with CRP and 6 edges with IL- 6 (Fig. 2A, second and third columns; Table S3A). The 6 edges negatively correlated with IL- 6 were a subset of the 21 edges significantly negatively correlated with CRP and were the previously highlighted DMN-VA intermodular edges which were anatomically localised between insular/frontal opercular and PCC (Fig. 2B).

We then averaged FC over all edges within the case-control mask to investigate how individual differences in mean FC within this network were related to inflammation. Average network connectivity was negatively correlated with CRP $(r=-0.41, P=0.00008)$ and IL-6 ( $r=$ $-0.36, P=0.0013$ ) (Fig. 2A, fourth column; Table S3B-C). These associations remained significant after adjusting for sex and BMI (Table S3BC). We also performed an additional sensitivity analysis to test the robustness of these relationships to the choice of NBS threshold ( $t_{\text {primary }}$ $=3.8$ ). The correlation was robust across three other primary thresholds $\left(\mathrm{t}_{\text {primary }}=3.1,3.3,3.5\right)$ (Fig. S6A-B).

\subsection{Association between FC and cellular markers in depression (Part 2)}

Analyzable cell count data were available on $\mathrm{N}=36$ depression cases, where neutrophils were significantly correlated with CRP $(r=$ $\left.0.57, P_{\mathrm{FDR}}<0.001\right)$ and IL-6 $\left(r=0.47, P_{\mathrm{FDR}}<0.05\right)$ (Fig. 3A, Table S1B). Neutrophils were also negatively correlated $(-0.41<\mathrm{r}<0)$ with functional connectivity of individual edges within case-control network mask, although none demonstrated significant association after FDR correction. Neutrophils scaled negatively $\left(\mathrm{r}=-0.34, P=0.025, P_{F D R}=\right.$ 0.05) against average network connectivity (Fig. S7; Table S3C). We observed a similar negative association for $\mathrm{CD} 4^{+} \mathrm{T}$-cells, although not significant (Fig. S7). Specificity analyses on the remaining 10 classes of immune cells revealed a negative trend towards significance in CD56 hi/ bright natural killer cells (Fig. S8).

We finally investigated differences in FC within the case-control mask between the cell-stratified subgroups of depression cases. Corroborating previous findings on a larger sample, the subset of highcell count $(\mathrm{N}=16)$ and low-cell count $(\mathrm{N}=20)$ depression groups also differed significantly in CRP, neutrophils, basophils, eosinophils, classical monocytes and NK T-cells (Table S1C). The high-cell count depression subgroup had significantly lower edge-wise distribution of FC than the low-cell count depression subgroup in the case-control network mask (KS test, $P=2.2 \times 10^{-16}, \mathrm{D}=0.11$, Cohen's $\mathrm{d}=0.12$ ) (Fig. 3B). Consistent with previous observations, negative edges were again concentrated between DMN-VA modules, with identical edges showing greatest negative correlation (Fig. 3C).

\section{Discussion}

As predicted by our first hypothesis, we found a network of interconnected edges with significantly reduced functional connectivity in depression cases with heightened peripheral inflammation (CRP $>3$ $\mathrm{mg} / \mathrm{L}$ ) compared to healthy controls. This NBS-derived case-control network comprised edges localized primarily to connections between DMN and VA functional modules, linking the left insular/frontalopercular and left posterior cingulate cortical regions anatomically. Group comparison of connectivity within this case-control network mask revealed a hierarchical increase in FC attenuation, with HC showing least impairment, followed by loCRP depression cases, and then hiCRP depression cases. These NBS findings corroborate and extend outcomes from our earlier study which analysed between-group FC differences on the same dataset (Kitzbichler et al., 2020).

Next, as predicted by our second hypothesis, we demonstrated negative scaling between CRP, IL-6, neutrophils and average network connectivity, within the depression cases only. Our analyses using cellstratified assignments into low- and high-cell count subgroups of depression, corroborated this observation in that more negative connections were noted in high-cell count compared to low-cell count subgroups, implicating identical connections to those stratified by CRP $i$. e. between insular/frontal opercular cortex and posterior cingulate cortex.

\subsection{Interoceptive network dysfunction in inflammation-linked depression}

More broadly, these results were consistent with evidence suggesting interoceptive dysfunction in inflammation-linked depression (Savitz and Harrison, 2018; Khalsa et al., 2018). Interoception is the perception of bodily physiological states - such as cardiovascular, gastrointestinal and immune systems - that has been deemed an important source of emotional experience (Craig, 2002). Interoceptive signalling i.e. the ability to "feel" what is happening within the body, is critical for emotional regulation, bodily homeostatic functioning and survival (Craig, 2002). In MDD, interoceptive dysfunction has been associated with reduced emotional experience i.e. "feeling nothing", alexithymia and anhedonia (Quadt et al., 2018). In inflammation-linked depression specifically, the interoceptive model provides some mechanistic insight 
A

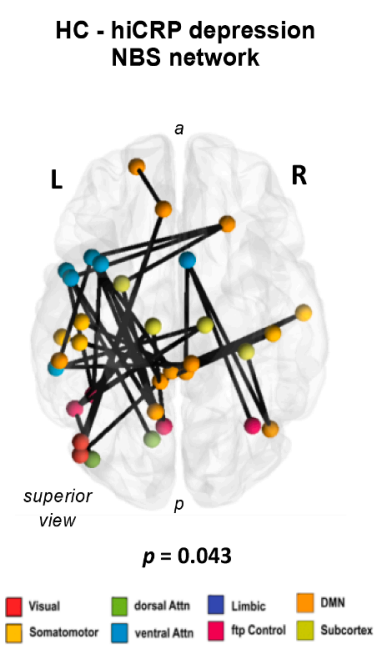

D

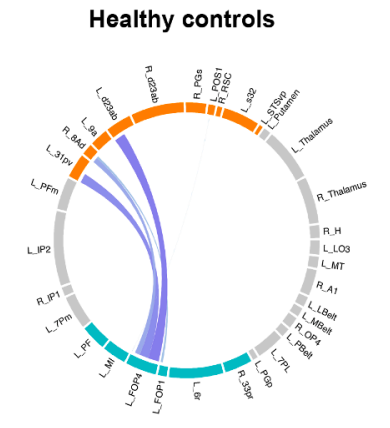

B Healthy controls

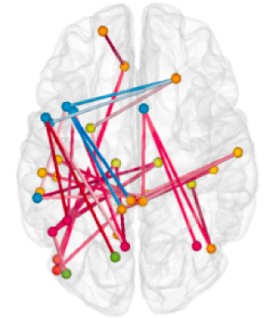

loCRP depression

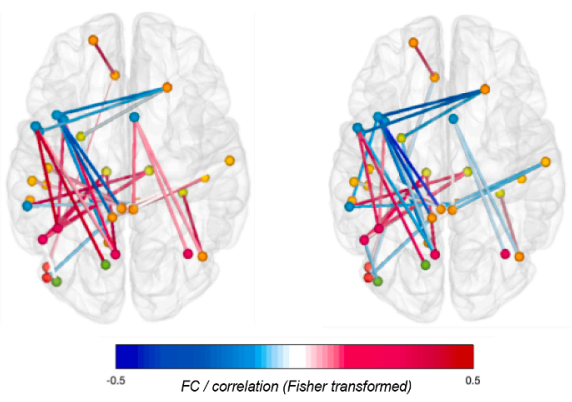

all depression

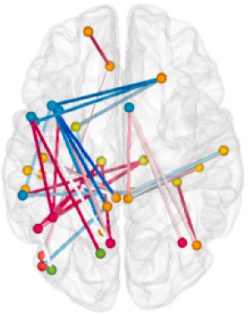

hiCRP depression
loCRP depression

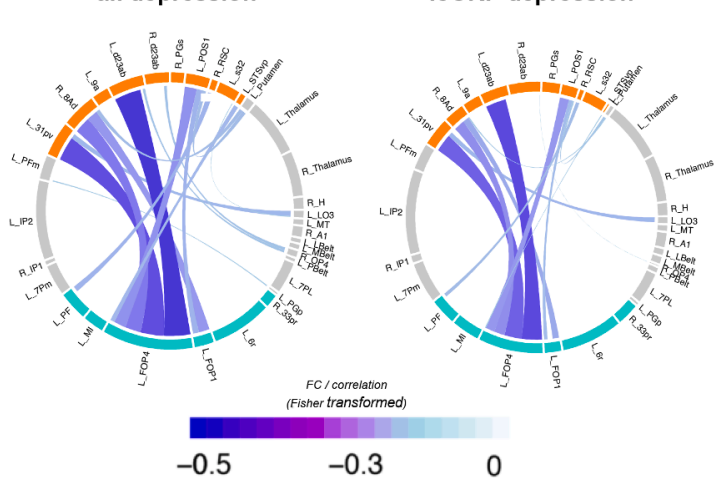

C

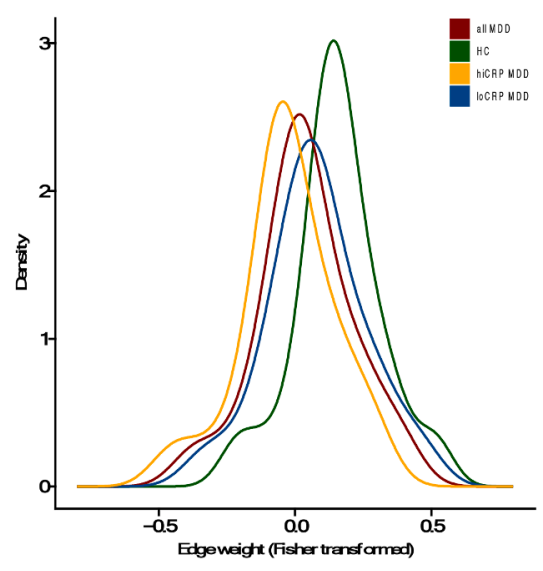

hiCRP depression

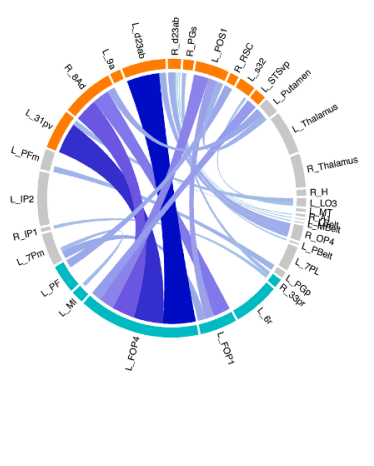

Fig. 1. Network-based case-control differences in functional connectivity. (A) Network of case-control differences in functional connectivity (HC vs hiCRP depression cases) identified by Network Based Statistics (NBS). A network comprising 38 edges or connections, between 33 nodes or regions, was generated by testing a onetailed hypothesis, i.e. $\mathrm{HC}>$ hiCRP cases, and reported here at the highest primary threshold, $\mathrm{t}_{\text {primary }}=3.8$, at which a significant $(\mathrm{P}<0.05)$ network was identifiable. Nodes (balls) are colour-coded according to their assignment to the 7 functional modules previously defined by Yeo et al. (2011) (Thomas Yeo et al., 2011). Anatomically, these connections mainly linked the insular/frontal-operculum and posterior cingulate cortical regions as defined by Glasser et al. (2016) anatomical area assignment, with constituent pairs of nodes also being assigned, respectively, to the default mode network (dark orange balls) and ventral attention (cyan balls) functional network (see Supplementary Data Section S3B). (B) Group-averaged functional connectivity across this case-control network, plotted for each group. Edges (sticks) are colour coded according to the sign and strength of functional connectivity (FC). Across HC, loCRP cases and hiCRP cases, reduced FC was observed (more blue connections or edges). (C) Distribution of edge-weights (or correlations) within the HC vs hiCRP cases NBS network per group. (D) Negatively-weighted edges within the HC vs hiCRP cases NBS network across groups, visualized in topological space via radial network diagram. The outer track is divided into sectors, each denoting a cortical node within the NBS network, labelled according to the nomenclature of the Glasser et al. (2016) cortical parcellation and coloured according to its assignment to one of 7 functional networks or modules as defined by Yeo et al., (2011) (Thomas Yeo et al., 2011) . Width of the sector denotes approximate weighted nodal degree (or number of connections per node / region) i.e. the greater the number of attenuated connections, the longer the sector. Thickness and colour of links denote the strength of functional connectivity (FC), where edges with more negative FC have thicker and bluer links. Links within each sector are ordered clockwise with increasing FC. Across HC, loCRP cases and hiCRP cases, increases in both number and weight of negative edges were observed, especially between nodes located in insula/frontal opercular cortex and functionally assigned to the ventral attention module, e.g., L_FOP4, and nodes located in the posterior cingulate cortex and assigned to the default mode network e.g., L_d23ab, L_31pv. Abbreviations are as follows: dorsal attn - dorsal attention network; ventral attn ventral attention network; DMN - default mode network; ftp control - frontoparietal control network. Highlighted brain regions or nodes in panel D : L_FOP4 - left frontal opercular area 4 (anatomical area = insula/frontal operculum; functional module = ventral attention); L_FOP1 - left frontal opercular area 1 (insula/frontal operculum; ventral attention); L/R_d23ab - left/right area dorsal $23 \mathrm{a}+\mathrm{b}$ (posterior cingulate cortex; default mode); L_31pv - left area 31pv (posterior cingulate cortex; default mode); L_STSvp - left area STSv posterior (dorsolateral prefrontal cortex; default mode); L_MI - left middle insular area (insula/frontal operculum; ventral attention); L_PF - area PF complex (insula/frontal operculum; ventral attention); L_33pr - left area 33 prime (insula/frontal operculum; ventral attention); L_6r - rostral area 6 (premotor cortex; ventral attention); L_POS1 - left parieto-occipital sulcus area 1(posterior cingulate cortex; default mode); L_9a - left area 9 anterior (dorsolateral prefrontal cortex; default mode); L_PF - left mid insula (insula/frontal operculum; ventral attention); R_8Ad - right area 8Ad (dorsolateral prefrontal cortex; default mode); R_RSC - right retrosplenial complex (posterior cingulate cortex; default mode). all depression - all depression cases; loCRP depression - low CRP depression cases; hiCRP depression - high CRP depression cases. (For interpretation of the references to colour in this figure legend, the reader is referred to the web version of this article.) 
A

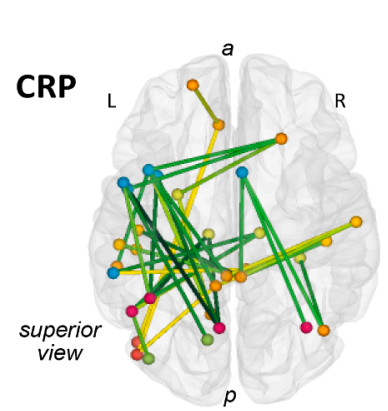

$\mathbf{L}$

IL-6
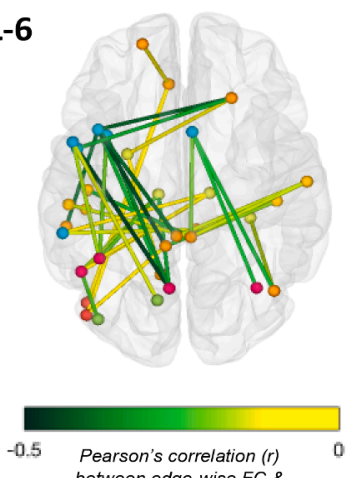
between edge-wise FC \& inflammatory biomarker

B
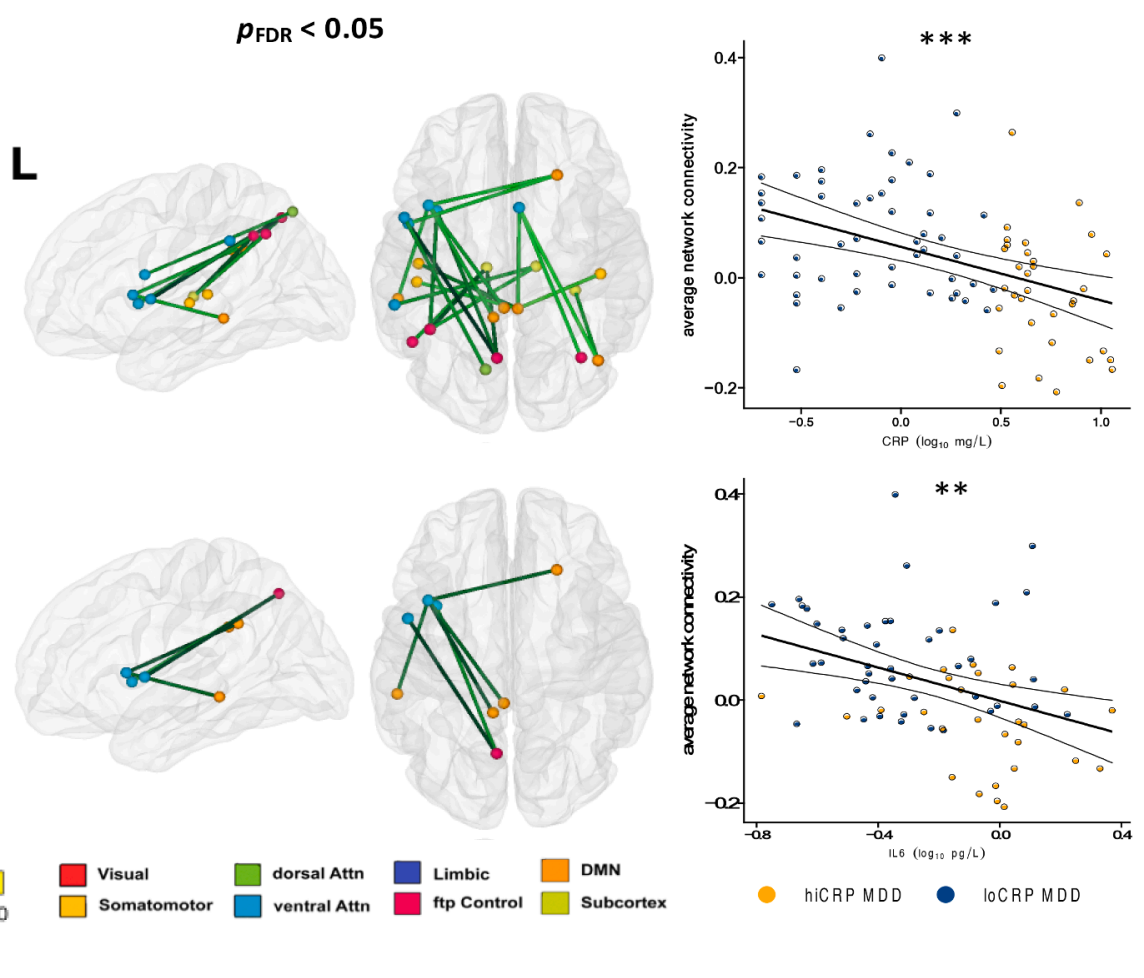
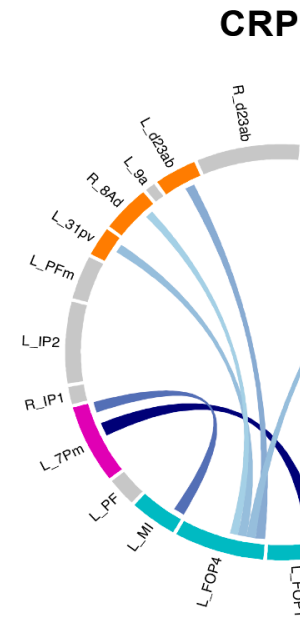

CRP

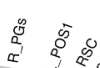

$\frac{10}{2}$

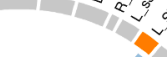

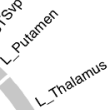
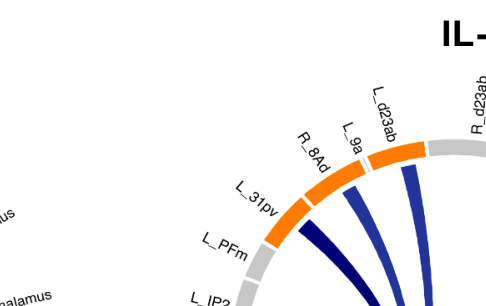

\section{IL-6}

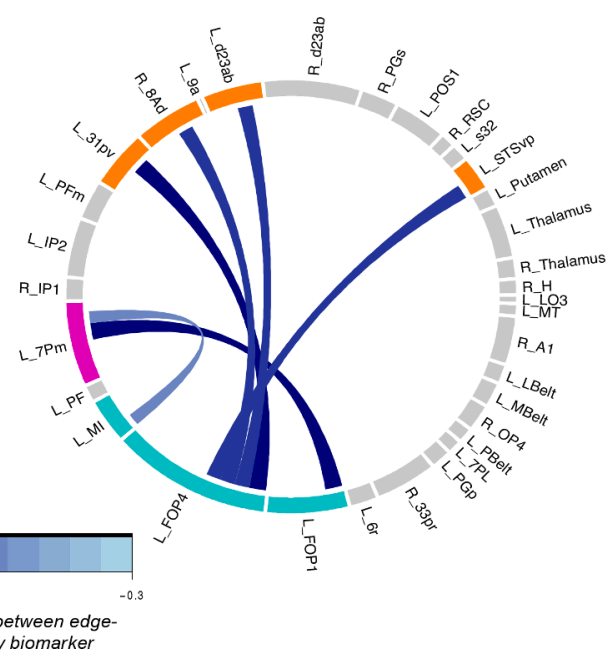

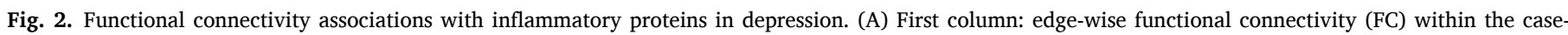

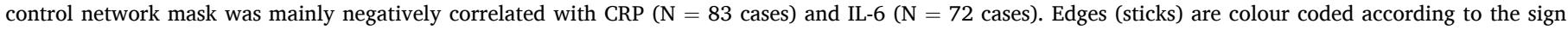

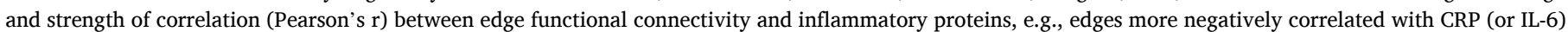

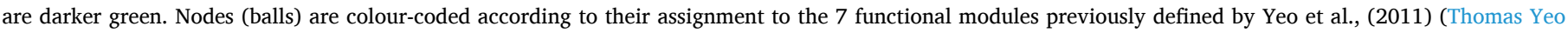

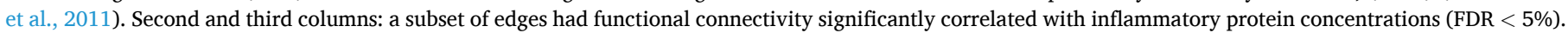

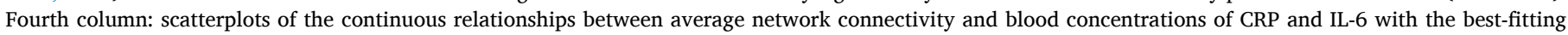

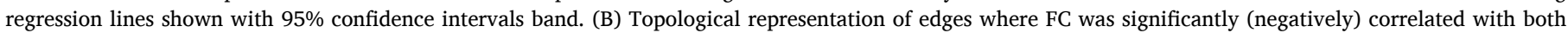

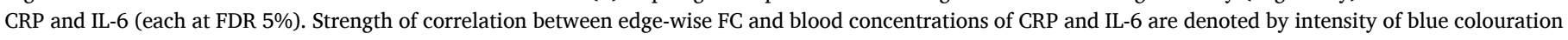

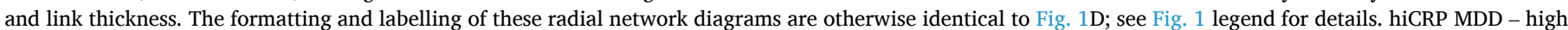

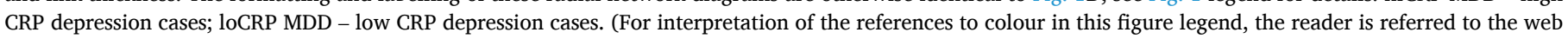
version of this article.)

by positing a role for bidirectional brain immune-signalling via neural pathways (predominantly the vagus nerve), that underpins communication of peripheral immune states to the brain and vice versa (Miller and Raison, 2016; Savitz and Harrison, 2018). Collectively described as the "interoceptive nervous system" (INS), the INS is thought to relay afferent vagal information from the medulla, to a brain system anchored in the insular, frontal opercular, cingulate and somatomotor cortices (Fischl et al., 2002; Zalesky et al., 2010; Zalesky et al., 2012; Cocchi et al., 2014; Xia et al., 2013). Regions within the INS are largely native to two canonical functional networks, namely the DMN and VA (also known as the salience network or cingulo-opercular network) (Kraynak et al., 2018; Critchley et al., 2004; Harshaw, 2015; Kleckner et al., 2017; 


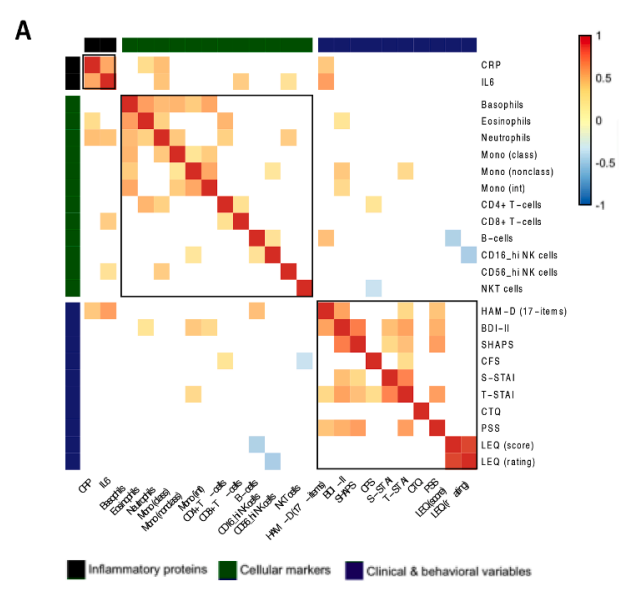

\section{B}

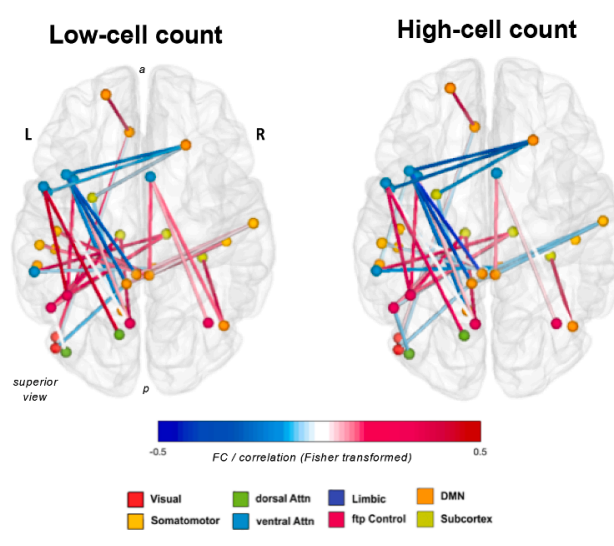

C

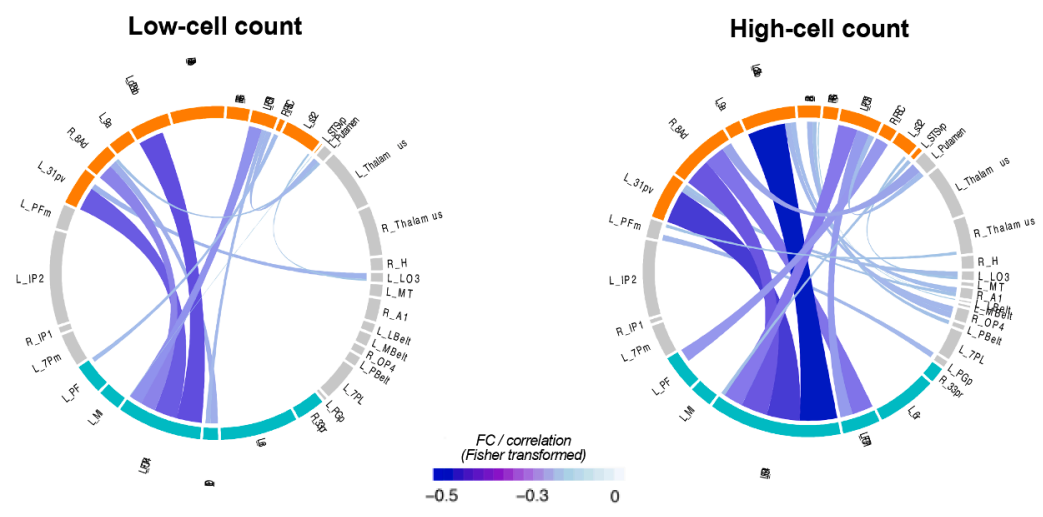

Fig. 3. Functional connectivity differences between high-cell count and lowcell count subgroups of depression. (A) Correlation matrix highlighting significant correlations between inflammatory proteins, absolute cell-counts and clinical variables thresholded at $\mathrm{P}<0.05$ ( $\mathrm{N}=36$ cases). (B) Group-averaged representation of functional connectivity within the NBS-derived case-control network mask for low-cell count and high-cell count subgroups of depression cases. These subgroup assignments were defined a priori following a Gaussian mixture model-based clustering analysis of absolute cell counts (12 immune cell classes) conducted in a previous study on a larger sample. Briefly, the high-cell count depression subgroup had increased absolute cell counts of neutrophils and other myeloid cells, and increased CRP compared to the low-cell count depression subgroup (see Supplementary Data Table S1C). (C) Negatively-weighted edges within the network mask for high-cell count and low-cell count subgroups represented in topological space. The high-cell count subgroup show a greater number of more attenuated edges, denoted by thicker and bluer links. The formatting and labelling of these radial network diagrams are identical to Fig. 1D; see Fig. 1 legend for details.
Critchley et al., 2003). We therefore interpreted our NBS-derived casecontrol network of attenuated FC in inflammation-linked depression, as indicative of disrupted function of the INS.

The concept of dysconnectivity (or disrupted connectivity) in interoceptive systems has been previously evidenced by human experimental models of inflammation-linked depression i.e. "sickness behaviour" following immune challenge (Kraynak et al., 2018). In particular, Dipasquale et al. (Dipasquale et al., 2016) applied NBS to investigate FC differences pre- and post-IFN $\alpha$ therapy in hepatitis-C positive participants (Dipasquale et al., 2016). This study identified a similar network comprising bilateral insula, frontal cortical and subcortical regions, showing reduced FC 4-hours after IFN $\alpha$ administration in participants. Interoceptive signalling has also been described to show domain specificity and hierarchical processing. Thus, higher-level neural processing of interoceptive signals could vary between afferent signal types e.g. affective (emotion), visceral physiology (immune, cardiac) or nociceptive (pain) inputs (Khalsa et al., 2018; Mai et al., 2019). As such, subsystems of the INS may exist. For example, interoception has also been linked to motivational circuit and could modulate reward sensitivity and hedonic sensing (Harrison et al., 2016; Critchley and Garfinkel, 2017). Attenuation within the corticostriatal and cortico-amygdalar pathways of the reward system has been reported with increased CRP in MDD (Sheline et al., 2009; Hamilton et al., 2015). A different study on the same dataset using PBNA (parcellation-based network-analysis) - a connectome-based technique similar to NBS (but using Bayesian multilevel modelling) - also demonstrated that increased CRP was again associated with decreased FC between a seeded region of ventromedial prefrontal cortical cluster and a network of cortical areas comprising classic INS regions e.g. insula and cingulate cortex (Yin et al., 2019). These prior reports are broadly convergent with our suggestion that "inflammation-sensitive" brain regions such as the insula and striatum are likely embedded within a subsystem of the INS that is critical for parsing immune and affective signals.

\subsection{Interoceptive immune-sensing dysfunction in inflammation-linked depression}

Findings from our secondary analyses, although less precedented, were also consistent with prior evidence. The negative scaling of FC against blood inflammatory markers corroborated reports from clinical MDD and population studies using a priori defined regions for functional connectivity analyses. For example, heightened peripheral CRP, IL-6, IL$1 \beta$ and IL-1Ra all negatively covaried with vmPFC-striatal and corticoamygdalar FC in MDD cases (Felger et al., 2016; Mehta et al., 2018). A population-based investigation then showed similar patterns of findings in that FC within an emotional regulation network negatively scaled with increasing inflammation composite score (aggregate of CRP, IL-6, IL-10 and TNF $\alpha$ ) and classical monocytes (Nusslock et al., 2019).

In addition to CRP and IL-6, our investigation of the relationship between absolute immune cell counts and FC showed further evidence for innate immune system involvement as the high-cell count depression subgroup had increased leukocytes of myeloid origin, especially neutrophils (Table S1C), and we noted significant negative scaling between neutrophil counts and FC. Nonetheless, it would be biased and perhaps premature to assert at this stage that only the innate immune system is central to the pathoetiology of inflammation-linked depression. Although neutrophils are widely accepted as primary effectors of the innate immune system and acute inflammation, these cells are not functionally exclusive to the innate immune system. Neutrophils engage heavily in immune cross-talk with resident cells of the adaptive immune system, particularly CD4 ${ }^{+}$T-cells, where mutual modulation of function occurs (Mantovani et al., 2011). For example, neutrophils are able to induce activation and promote differentiation of naïve $\mathrm{CD} 4^{+} \mathrm{T}$-cells. Reciprocally, regulatory T-cells produce cytokines promoting survival of neutrophils, that otherwise undergo frequent spontaneous apoptosis to facilitate normal cell turnover (Kalyan and Kabelitz, 2014; Li et al., 2019). We also noted from our specificity analyses that CD $56^{\text {hi/bright }}$ 
natural killer (NK) cells showed an identical (but not significant) pattern of association with FC (Fig. S8). CD56 hi/bright NK cells are one of the key effector cells of the innate immune system, although of lymphoid origin and typically reside in lymph nodes where they have been reported to interact with $\mathrm{CD}^{+}{ }^{+} \mathrm{T}$-cells via cytokine signalling (Fehniger et al., 2003; Vivier et al., 2008; Poli et al., 2009; Gabrielli et al., 2016). Thus, it is plausible that NK cells (and other immune cell classes besides neutrophils and $\mathrm{CD} 4^{+} \mathrm{T}$ cells) may be associated with changes in brain functional connectivity in inflammation-related depression. Future studies with larger sample size may be expected to detect these associations more powerfully.

In the context of inflammation-linked depression, our findings suggest that the brain-immune relationship involves interoceptive immunesensing or perception of internal immune state by the brain. This proposition is consistent with evidence supporting the "immunological homunculus" (Tracey, 2007; Diamond and Tracey, 2011; Schiller et al., 2021) - the concept that discrete neural networks coordinate components of the peripheral immune system via the cholinergic antiinflammatory pathway, and rostro-caudal functional gradients exhibited by the insula. In the human interoceptive model, the posterior, middle and anterior parts of the insular cortex are thought to play different roles. Lower-level (peripheral) sensory information is firstly encoded in the posterior insula, before being represented in the midinsula where convergent signals, e.g. hedonic/motivational signals to ascribe salience, are integrated with other sensory brain regions. This information is then relayed to the anterior insula, where in conjunction with cingulate cortex, behavioural responses and emotional changes are elicited (Craig, 2002; (Bud) Craig, 2009; Craig, 2009). Thus the posterior insula may be viewed as the immune sensory cortex, whereas the anterior insula is linked to higher-order emotion regulation, such as subjective feeling states of "sickness" and/or "sadness", and related depressive states such as anhedonia (Namkung et al., 2017; Lekander et al., 2016). Hence, we interpreted our observations as evidence of dysconnectivity within the INS associated with impairment of immunesensing and effects on mood regulation in depression.

\subsection{Strengths and limitations}

A strength of our study was the use of NBS for whole-brain analysis of FC abnormalities in inflammation-linked depression. Comparable prior studies have generally used a priori defined brain regions and canonical functional networks to ascertain FC alterations. In contrast, NBS allowed us to perform an unbiased whole-brain investigation beyond the constraints of canonical functional networks, which is a more robust approach to mapping effects of inflammation in depression, as networks, clusters and circuits within the brain are more likely to be affected than single isolated connections or regions. Additionally, we used the Glasser parcellation which is currently the highest definition parcellation scheme for the insular-opercular region, delineating 13 insular/frontalopercular subdivisions on the basis of a combination of features derived from multiple imaging modalities (Uddin et al., 2017). Our study also presents FC associations with immune cell markers, that have not been widely considered in its relationship to brain functional connectivity, compared to inflammatory proteins such as CRP and IL-6.

An important limitation of our study is the lack of high CRP $(>3 \mathrm{mg}$ ) L) controls. In future, it will be important also to analyse differences between depression cases and non-depression controls, with CRP levels allowed to exceed the $3 \mathrm{mg} / \mathrm{L}$ cut-off in both groups, to determine if functional connectivity of the interoceptive network is correlated with peripheral inflammation in a non-depression population, e.g., using population derived data such as the UK Biobank cohort. It is also important to clarify that although we interpreted our results in relation to prior knowledge of interoceptive systems, we are not asserting that the brain regions identified by the NBS analysis of hiCRP case-control differences are linked exclusively to interoception. Several studies have highlighted the anterior insula as part of a "multiple-demand" system or network (Dosenbach et al., 2006; Nelson et al., 2010; Simmons et al., 2013) and, together with the anterior cingulate and frontal cortex, the insula/operculum has been posited to form a "core" task-dependent brain network responsible for encoding error signals and sustaining attentional control (Dosenbach et al., 2006). Therefore, more work is needed to strengthen our claim regarding the sensitivity of interoceptive networks to peripheral inflammatory signals, as suggested by these data.

Although the sample size was consistent with many prior studies, it was somewhat underpowered to detect subtle associations between FC and peripheral inflammation. Sampling bias was evident in terms of a greater proportion of females, particularly in the hiCRP depression subgroup, although the cases (overall) and controls were prospectively matched for age and sex. Unsurprisingly in this context, case-control differences were not significant in the Part 1 sensitivity analyses that controlled for sex and BMI by prior regression of these covariates on the FC matrices before NBS analysis (Fig. S5B). However, the impact of controlling for sex or BMI was less marked in the Part 2 sensitivity analyses. All significant relationships between FC and immune biomarkers within the group of all depression cases were conserved, albeit with smaller effect sizes, when sex was included as a covariate in the linear model (Table S3B-D), or when within-group analysis was restricted to female cases only (Fig. S6B).

Fundamentally, in a cross-sectional design like this, it is difficult to disentangle the potential moderating or confounding effects of adiposity on inflammation and functional connectivity related to depression. Ideally, this will require further investigation with longitudinal designs and non-depression control groups representing a wider range of variation in BMI and inflammatory biomarkers. It is well known that BMI is positively correlated with CRP, as it was in these data $(r=0.47)$. However, it is not so clear how BMI should be handled statistically in analysis of the relationships between depression, functional connectivity, and CRP or other inflammatory biomarkers. Depression is associated with higher BMI and, indeed, obesity has been regarded as an aspect of the atypical depression syndrome characterized by hyperphagia and increased risk of metabolic disorders (Milaneschi et al., 2020; Lamers et al., 2020). On this assumption i.e. BMI is an integral part of the depressive phenotype, then it should not be controlled for statistically in the analysis of depression-related differences in functional connectivity, which is the principal analytic approach we adopted for both parts of this investigation. However, the contrary view is that BMI is a confounding variable, rather than integral to depression, and it should be statistically controlled as a "nuisance covariate", which is the approach we adopted for the sensitivity analyses in Parts 1 and 2 . We found that the within-group analyses of association between inflammatory biomarkers and functional connectivity remained significant after controlling for BMI; whereas the between-group analysis of FC differences between hiCRP depression cases and healthy controls was more sensitive to statistical correction for BMI. In short, our findings do show some support for an "immunometabolic" subtype of depression (or atypical depression) (Milaneschi et al., 2020; Lamers et al., 2020) that comprises metabolic and immune dysregulation e.g. obesity and heightened peripheral inflammation as part of the clinical symptomatology.

In relation to immune biomarkers, we note that we only had access to data on one cytokine (IL-6) for the purpose of this analysis. Although IL6 has been widely implicated in previous studies of depression, we cannot conclude that it is the only cytokine associated with changes in brain functional connectivity. It will be important to include a wider range of cytokines in future fMRI studies of inflammation-related depression. Finally, we also note that our observations were based on cross-sectional investigation (and not longitudinal), limiting causal interpretation at present.

\section{Conclusions}

While the relevance of interoception to inflammation-linked depression has been previously discussed, this study provides direct 
evidence for an association between increased protein and cellular biomarkers of inflammation, and decreased functional connectivity in an interoceptive brain network identified by whole-brain analysis in a depression sample. These results point towards a putative etiological model of inflammation-linked depression, where peripheral inflammation is linked to dysconnectivity of a brain network specialised in peripheral immune sensing and emotion regulation.

\section{Article information}

\section{Disclosures}

ETB serves on the advisory board of Sosei Heptares and as a consultant for GlaxoSmithKline. All other authors declare no competing interests.

\section{Funding}

This study was funded by an award from the Wellcome Trust (grant number: 104025/Z/14/Z) for the Neuroimmunology of Mood Disorders and Alzheimer's Disease (NIMA) Consortium in partnership with Janssen, GlaxoSmithKline, Lundbeck and Pfizer. Additional support was provided by the National Institute for Health Research (NIHR) Biomedical Research Centre (BRC) at Cambridge (Mental Health and Cell Phenotyping Hub), the NIHR BRC at South London and Maudsley NHS Foundation Trust and King's College London, and an NIHR Senior Investigator award (to ETB).

\section{Data availability}

Sharing of data used in this study on public repositories is restricted by the informed consent process. However, in compliance with the Wellcome Trust open research policy, part of the research data is being made available on an institutional restricted access repository (IDE-CAM Repository; https://portal.ide-cam.org.uk/collections), where access could be granted on request pending approval by Principal Investigator (Professor Edward Bullmore) and/or Corresponding Author, to accredited research groups. Further information and request for code should be directed to Corresponding Author.

\section{Acknowledgements}

The authors thank all the participants in the study and members of the NIMA research team - in particular, project coordinators Linda Pointon, Junaid Bhatti, Ciara O'Donnell (see Supplementary Appendix for a complete list of NIMA Consortium members), medical imaging staff and laboratory staff. František Vàša provided valuable feedback on an earlier version of the manuscript.

\section{Appendix A. Supplementary data}

Supplementary data to this article can be found online at https://doi. org/10.1016/j.bbi.2021.08.226.

\section{References}

Schmaal, L., Pozzi, E., Ho, T., van Velzen, L.S., Veer, I.M., Opel, N., Van Someren, E.J.W. Han, L.K.M., Aftanas, L., Aleman, A., Baune, B.T., Berger, K., Blanken, T.F., Capitão, L., Couvy-Duchesne, B., Cullen, K.R., Dannlowski, U., Davey, C., ErwinGrabner, T., Evans, J., Frodl, T., Fu, C.H.Y., Godlewska, B., Gotlib, I.H., GoyaMaldonado, R., Grabe, H.J., Groenewold, N.A., Grotegerd, D., Gruber, O., Gutman, B.A., Hall, G.B., Harrison, B.J., Hatton, S.N., Hermesdorf, M., Hickie, I.B., Hilland, E., Irungu, B., Jonassen, R., Kelly, S., Kircher, T., Klimes-Dougan, B., Krug, A., Landrø, N.I., Lagopoulos, J., Leerssen, J., Li, M., Linden, D.E.J., MacMaster, F.P., McIntosh, A., Mehler, D.M.A., Nenadić, I., Penninx, B.W.J.H., Portella, M.J., Reneman, L., Rentería, M.E., Sacchet, M.D., Sämann, P., Schrantee, A., Sim, K., Soares, J.C., Stein, D.J., Tozzi, L., van Der Wee, N.J.A., van Tol, M.-J., Vermeiren, R., Vives-Gilabert, Y., Walter, H., Walter, M., Whalley, H.C., Wittfeld, K., Whittle, S., Wright, M.J., Yang, T.T., Zarate, C., Thomopoulos, S.I., Jahanshad, N.,
Thompson, P.M., Veltman, D.J., 2020. ENIGMA MDD: seven years of global neuroimaging studies of major depression through worldwide data sharing. Translational. Psychiatry 10 (1). https://doi.org/10.1038/s41398-020-0842-6.

Kiecolt-Glaser, J.K., Derry, H.M., Fagundes, C.P., 2015. Inflammation: depression fans the flames and feasts on the heat. AJP 172 (11), 1075-1091.

Otte, C., Gold, S.M., Penninx, B.W., Pariante, C.M., Etkin, A., Fava, M., Mohr, D.C., Schatzberg, A.F., 2016. Major depressive disorder. Nat. Rev. Dis. Primers 2 (1). https://doi.org/10.1038/nrdp.2016.65.

Maes, M., Van Der Planken, M., Stevens, W.J., Peeters, D., DeClerck, L.S., Bridts, C.H., Schotte, C., Cosyns, P., 1992. Leukocytosis, monocytosis and neutrophilia: hallmarks of severe depression. J Psychiatr Res 26 (2), 125-134.

Dantzer, R., O'Connor, J.C., Freund, G.G., Johnson, R.W., Kelley, K.W., 2008. From inflammation to sickness and depression: when the immune system subjugates the brain. Nat Rev Neurosci 9 (1), 46-56.

Miller, A.H., Raison, C.L., 2016. The role of inflammation in depression: from evolutionary imperative to modern treatment target. Nat. Rev. Immunol. 16 (1), 22-34.

Khandaker, G.M., Dantzer, R., Jones, P.B., 2017. Immunopsychiatry: important facts. Psychol. Med. 47 (13), 2229-2237.

Pariante, C.M., 2015. Psychoneuroimmunology or immunopsychiatry? The Lancet Psychiatry 2 (3), 197-199.

Khandaker, G., Harrison, N., Bullmore, E. (Eds.), 2021. Textbook of Immunopsychiatry [Internet]. Cambridge University Press, Cambridge.

Haapakoski, R., Mathieu, J., Ebmeier, K.P., Alenius, H., Kivimäki, M., 2015. Cumulative meta-analysis of interleukins 6 and $1 \beta$, tumour necrosis factor $\alpha$ and C-reactive protein in patients with major depressive disorder. Brain Behav. Immun. 49, 206-215.

Osimo, E.F., Pillinger, T., Rodriguez, I.M., Khandaker, G.M., Pariante, C.M., Howes, O.D., 2020. Inflammatory markers in depression: a meta-analysis of mean differences and variability in 5,166 patients and 5,083 controls [Internet]. Brain Behav. Immun. 87, 901-909.

Lynall, M.-E., Turner, L., Bhatti, J., Cavanagh, J., de Boer, P., Mondelli, V., Jones, D., Drevets, W.C., Cowen, P., Harrison, N.A., Pariante, C.M., Pointon, L., Clatworthy, M. R., Bullmore, E., 2020. Peripheral blood cell-stratified subgroups of inflamed depression. Biol. Psychiatry 88 (2), 185-196.

Khandaker, G.M., Pearson, R.M., Zammit, S., Lewis, G., Jones, P.B., 2014. Association of serum interleukin 6 and C-reactive protein in childhood with depression and psychosis in young adult life: a population-based longitudinal study. JAMA Psychiatry 71 (10), 1121. https://doi.org/10.1001/jamapsychiatry.2014.1332.

Nusslock, R., Miller, G.E., 2016. Early-life adversity and physical and emotional health across the lifespan: a neuroimmune network hypothesis. Biol. Psychiatry 80 (1), 23-32.

Wohleb, E.S., Franklin, T., Iwata, M., Duman, R.S., 2016. Integrating neuroimmune systems in the neurobiology of depression. Nat. Rev. Neurosci. 17 (8), 497-511.

Logothetis, N.K., 2008. What we can do and what we cannot do with fMRI. Nature 453 (7197), 869-878.

Fox, M.D., Greicius, M., 2010. Clinical applications of resting state functional connectivity [Internet]. Front. Syst. Neurosci.

Thomas Yeo, B.T., Krienen, F.M., Sepulcre, J., Sabuncu, M.R., Lashkari, D., Hollinshead, M., Roffman, J.L., Smoller, J.W., Zöllei, L., Polimeni, J.R., Fischl, B., Liu, H., Buckner, R.L., 2011. The organization of the human cerebral cortex estimated by intrinsic functional connectivity. J. Neurophysiol. 106 (3), 1125-1165.

Sheline, Y.I., Barch, D.M., Price, J.L., Rundle, M.M., Vaishnavi, S.N., Snyder, A.Z., Mintun, M.A., Wang, S., Coalson, R.S., Raichle, M.E., 2009. The default mode network and self-referential processes in depression. PNAS 106 (6), 1942-1947.

Hamilton, J.P., Farmer, M., Fogelman, P., Gotlib, I.H., 2015. Depressive rumination, the default-mode network, and the dark matter of clinical neuroscience. Biol. Psychiatry 78 (4), 224-230.

Seeley, W.W., 2019. The salience network: a neural system for perceiving and responding to homeostatic demands. J Neurosci 39 (50), 9878-9882.

Kaiser, R.H., Andrews-Hanna, J.R., Wager, T.D., Pizzagalli, D.A., 2015. Large-scale network dysfunction in major depressive disorder: a meta-analysis of resting-state functional connectivity. JAMA Psychiatry 72 (6), 603. https://doi.org/10.1001/ jamapsychiatry.2015.0071.

Kraynak, T.E., Marsland, A.L., Wager, T.D., Gianaros, P.J., 2018. Functional neuroanatomy of peripheral inflammatory physiology: a meta-analysis of human neuroimaging studies. Neurosci. Biobehav. Rev. 94, 76-92.

Felger, J.C., Li, Z., Haroon, E., Woolwine, B.J., Jung, M.Y., Hu, X., Miller, A.H., 2016. Inflammation is associated with decreased functional connectivity within corticostriatal reward circuitry in depression. Mol. Psychiatry 21 (10), 1358-1365.

Mehta, N.D., Haroon, E., Xu, X., Woolwine, B.J., Li, Z., Felger, J.C., 2018. Inflammation negatively correlates with amygdala-ventromedial prefrontal functional connectivity in association with anxiety in patients with depression: preliminary results [Internet]. Brain Behav. Immun. 73, 725-730.

Yin, L., Xu, X., Chen, G., Mehta, N.D., Haroon, E., Miller, A.H., Luo, Y., Li, Z., Felger, J.C., 2019. Inflammation and decreased functional connectivity in a widely-distributed network in depression: Centralized effects in the ventral medial prefrontal cortex. Brain Behav. Immun. 80, 657-666.

Damasio, A.R., Grabowski, T.J., Bechara, A., Damasio, H., Ponto, L.L.B., Parvizi, J., Hichwa, R.D., 2000. Subcortical and cortical brain activity during the feeling of selfgenerated emotions. Nat. Neurosci. 3 (10), 1049-1056.

Critchley, H.D., Garfinkel, S.N., 2017. Interoception and emotion. Curr. Opin. Psychol. $17,7-14$.

Savitz, J., Harrison, N.A., 2018. Interoception and Inflammation in Psychiatric Disorders. Biol Psychiatry Cogn Neurosci Neuroimaging 3 (6), 514-524. 
Kitzbichler, M.G., Aruldass, A.R., Barker, G.J., et al., Peripheral inflammation is associated with micro-structural and functional connectivity changes in depressionrelated brain networks. medRxiv 2020; 2020.09.09.20191262.

Veer, I.M., Beckmann, C., Van Tol, M.-J., et al., 2010. Whole brain resting-state analysis reveals decreased functional connectivity in major depression [Internet]. Front. Syst. Neurosci.

Pearson, T.A., Mensah, G.A., Alexander, R.W., Anderson, J.L., Cannon, R.O., Criqui, M., Fadl, Y.Y., Fortmann, S.P., Hong, Y., Myers, G.L., Rifai, N., Smith, S.C., Taubert, K., Tracy, R.P., Vinicor, F., 2003. Markers of inflammation and cardiovascular disease. Circulation 107 (3), 499-511.

Wium-Andersen, M.K., Ørsted, D.D., Nielsen, S.F., et al., 2013. Elevated C-reactive protein levels, psychological distress, and depression in 73131 individuals. JAMA Psychiatry 70, 176-184.

Raison, C.L., Rutherford, R.E., Woolwine, B.J., et al., 2013. A randomized controlled trial of the tumor necrosis factor-alpha antagonist infliximab in treatment resistant depression: role of baseline inflammatory biomarkers. JAMA Psychiatry 70, 31-41.

Uher, R., Tansey, K.E., Dew, T., Maier, W., Mors, O., Hauser, J., Dernovsek, M.Z., Henigsberg, N., Souery, D., Farmer, A., McGuffin, P., 2014. An inflammatory biomarker as a differential predictor of outcome of depression treatment with escitalopram and nortriptyline. AJP 171 (12), 1278-1286.

Mantovani, A., Cassatella, M.A., Costantini, C., Jaillon, S., 2011. Neutrophils in the activation and regulation of innate and adaptive immunity. Nat. Rev. Immunol. 11 (8), 519-531.

Glimcher, L.H., Murphy, K.M., 2000. Lineage commitment in the immune system: the T helper lymphocyte grows up. Genes Dev 14, 1693-1711.

Kundu, P., Inati, S.J., Evans, J.W., Luh, W.-M., Bandettini, P.A., 2012. Differentiating BOLD and Non-BOLD signals in fMRI time series using multi-echo EPI. Neuroimage 60 (3), 1759-1770.

Kundu, P., Brenowitz, N.D., Voon, V., Worbe, Y., Vertes, P.E., Inati, S.J., Saad, Z.S., Bandettini, P.A., Bullmore, E.T., 2013. Integrated strategy for improving functional connectivity mapping using multiecho fMRI. PNAS 110 (40), 16187-16192.

Glasser, M.F., Coalson, T.S., Robinson, E.C., Hacker, C.D., Harwell, J., Yacoub, E., Ugurbil, K., Andersson, J., Beckmann, C.F., Jenkinson, M., Smith, S.M., Van Essen, D. C., 2016. A multi-modal parcellation of human cerebral cortex. Nature 536 (7615), 171-178.

Fischl, B., Salat, D.H., Busa, E., Albert, M., Dieterich, M., Haselgrove, C., van der Kouwe, A., Killiany, R., Kennedy, D., Klaveness, S., Montillo, A., Makris, N., Rosen, B., Dale, A.M., 2002. Whole brain segmentation: automated labeling of neuroanatomical structures in the human brain. Neuron 33 (3), 341-355.

Fischl, B., 2012. FreeSurfer. Neuroimage 62 (2), 774-781.

Zalesky, A., Fornito, A., Bullmore, E.T., 2010. Network-based statistic: identifying differences in brain networks. NeuroImage 53 (4), 1197-1207.

Zalesky, A., Cocchi, L., Fornito, A., Murray, M.M., Bullmore, E.d., 2012. Connectivity differences in brain networks. NeuroImage 60 (2), 1055-1062.

Cocchi, L., Harding, I.H., Lord, A., Pantelis, C., Yucel, M., Zalesky, A., 2014. Disruption of structure-function coupling in the schizophrenia connectome. Neuroimage Clin 4, 779-787.

Xia, M., Wang, J., He, Y., Csermely, P., 2013. BrainNet viewer: a network visualization tool for human brain connectomics. PLoS ONE 8 (7), e68910.

Fox, P.T., Lancaster, J.L., 2002. Mapping context and content: the BrainMap model. Nat. Rev. Neurosci. 3 (4), 319-321.

Damasio, A., Carvalho, G.B., 2013. The nature of feelings: evolutionary and neurobiological origins. Nat. Rev. Neurosci. 14 (2), 143-152.

Khalsa, S.S., Adolphs, R., Cameron, O.G., Critchley, H.D., Davenport, P.W., Feinstein, J. S., Feusner, J.D., Garfinkel, S.N., Lane, R.D., Mehling, W.E., Meuret, A.E., Nemeroff, C.B., Oppenheimer, S., Petzschner, F.H., Pollatos, O., Rhudy, J.L., Schramm, L.P., Simmons, W.K., Stein, M.B., Stephan, K.E., Van den Bergh, O., Van Diest, I., von Leupoldt, A., Paulus, M.P., Ainley, V., Al Zoubi, O., Aupperle, R., Avery, J., Baxter, L., Benke, C., Berner, L., Bodurka, J., Breese, E., Brown, T., Burrows, K., Cha, Y.-H., Clausen, A., Cosgrove, K., Deville, D., Duncan, L., Duquette, P., Ekhtiari, H., Fine, T., Ford, B., Garcia Cordero, I., Gleghorn, D., Guereca, Y., Harrison, N.A., Hassanpour, M., Hechler, T., Heller, A., Hellman, N., Herbert, B., Jarrahi, B., Kerr, K., Kirlic, N., Klabunde, M., Kraynak, T., Kriegsman, M., Kroll, J., Kuplicki, R., Lapidus, R., Le, T., Hagen, K.L., Mayeli, A., Morris, A., Naqvi, N., Oldroyd, K., Pané-Farré, C., Phillips, R., Poppa, T., Potter, W. Puhl, M., Safron, A., Sala, M., Savitz, J., Saxon, H., Schoenhals, W., StanwellSmith, C., Teed, A., Terasawa, Y., Thompson, K., Toups, M., Umeda, S., Upshaw, V., Victor, T., Wierenga, C., Wohlrab, C., Yeh, H.-W., Yoris, A., Zeidan, F., Zotev, V., Zucker, N., 2018. Interoception and mental health: a roadmap. Biol Psychiatry Cogn Neurosci Neuroimaging 3 (6), 501-513.

Craig, A.D., 2002. How do you feel? Interoception: the sense of the physiological condition of the body. Nat. Rev. Neurosci. 3 (8), 655-666.

Quadt, L., Critchley, H.D., Garfinkel, S.N., 2018. The neurobiology of interoception in health and disease. Ann. N. Y. Acad. Sci. 1428, 112-128.

Critchley, H.D., Mathias, C.J., Josephs, O., et al., Human cingulate cortex and autonomic control: converging neuroimaging and clinical evidence. Brain 2003; 126: 2139-2152.
Critchley, H.D., Wiens, S., Rotshtein, P., Öhman, A., Dolan, R.J., 2004. Neural systems supporting interoceptive awareness. Nat. Neurosci. 7 (2), 189-195.

Harshaw, C., 2015. Interoceptive dysfunction: toward an integrated framework for understanding somatic and affective disturbance in depression. Psychol. Bull. 141 (2), 311-363.

Kleckner, I.R., Zhang, J., Touroutoglou, A., Chanes, L., Xia, C., Simmons, W.K., Quigley, K.S., Dickerson, B.C., Feldman Barrett, L., 2017. Evidence for a large-scale brain system supporting allostasis and interoception in humans. Nat. Hum. Behav. 1 (5) https://doi.org/10.1038/s41562-017-0069.

Dipasquale, O., Cooper, E.A., Tibble, J., Voon, V., Baglio, F., Baselli, G., Cercignani, M., Harrison, N.A., 2016. Interferon- $\alpha$ acutely impairs whole-brain functional connectivity network architecture - A preliminary study. Brain Behav Immun 58, 31-39.

Mai, S., Braun, J., Probst, V., Kammer, T., Pollatos, O., 2019. Changes in emotional processing following interoceptive network stimulation with rTMS. Neuroscience 406, 405-419.

Harrison, N.A., Voon, V., Cercignani, M., Cooper, E.A., Pessiglione, M., Critchley, H.D., 2016. A neurocomputational account of how inflammation enhances sensitivity to punishments versus rewards. Biol. Psychiatry 80 (1), 73-81.

Nusslock, R., Brody, G.H., Armstrong, C.C., Carroll, A.L., Sweet, L.H., Yu, T., Barton, A. W., Hallowell, E.S., Chen, E., Higgins, J.P., Parrish, T.B., Wang, L., Miller, G.E., 2019. SuppM_Higher peripheral inflammatory signaling associated with lower resting-state functional brain connectivity in emotion regulation and central executive networks. Biol. Psychiatry 86 (2), 153-162.

Kalyan, S., Kabelitz, D., 2014. When neutrophils meet T cells: beginnings of a tumultuous relationship with underappreciated potential. Eur. J. Immunol. 44 (3), 627-633.

Li, Y., Wang, W., Yang, F., Xu, Y., Feng, C., Zhao, Y., 2019. The regulatory roles of neutrophils in adaptive immunity. Cell Commun. Signaling 17 (1). https://doi.org/ 10.1186/s12964-019-0471-y.

Fehniger, T.A., Cooper, M.A., Nuovo, G.J., Cella, M., Facchetti, F., Colonna, M., Caligiuri, M.A., 2003. CD56bright natural killer cells are present in human lymph nodes and are activated by T cell-derived IL-2: a potential new link between adaptive and innate immunity. Blood 101 (8), 3052-3057.

Vivier, E., Tomasello, E., Baratin, M., Walzer, T., Ugolini, S., 2008. Functions of natural killer cells. Nat. Immunol. 9 (5), 503-510.

Poli, A., Michel, T., Thérésine, M., et al., CD56bright natural killer (NK) cells: an important NK cell subset. Immunology 2009; 126:458-465.

Gabrielli, S., Ortolani, C., del Zotto, G., Luchetti, F., Canonico, B., Buccella, F., Artico, M., Papa, S., Zamai, L., 2016. The memories of NK cells: innate-adaptive immune intrinsic crosstalk [Internet]. J. Immunol. Res. 2016, 1-14.

Tracey, K.J., 2007. Physiology and immunology of the cholinergic antiinflammatory pathway. J. Clin. Invest. 117 (2), 289-296.

Diamond, B., Tracey, K.J., 2011. Mapping the immunological homunculus. PNAS 108 (9), 3461-3462.

Schiller, M., Ben-Shaanan, T.L., Rolls, A., 2021. Neuronal regulation of immunity: why, how and where? Nat. Rev. Immunol. 21 (1), 20-36.

(Bud) Craig, A.D., 2009. How do you feel - now? The anterior insula and human awareness. Nat. Rev. Neurosci. 10 (1), 59-70.

Craig, A.D.(., 2009. (Bud): Emotional moments across time: a possible neural basis for time perception in the anterior insula. Philos Trans R Soc Lond B Biol Sci 364 (1525), 1933-1942.

Namkung, H.o., Kim, S.-H., Sawa, A., 2017. The insula: an underestimated brain area in clinical neuroscience, psychiatry, and neurology. Trends Neurosci 40 (4), 200-207.

Lekander, M., Karshikoff, B., Johansson, E., Soop, A., Fransson, P., Lundström, J.N., Andreasson, A., Ingvar, M., Petrovic, P., Axelsson, J., Nilsonne, G., 2016. Intrinsic functional connectivity of insular cortex and symptoms of sickness during acute experimental inflammation. Brain Behav. Immun. 56, 34-41.

Uddin, L.Q., Nomi, J.S., Hébert-Seropian, B., Ghaziri, J., Boucher, O., 2017. Structure and function of the human insula. J. Clin. Neurophysiol. 34 (4), 300-306.

Simmons, W.K., Avery, J.A., Barcalow, J.C., Bodurka, J., Drevets, W.C., Bellgowan, P., 2013. Keeping the body in mind: Insula functional organization and functional connectivity integrate interoceptive, exteroceptive, and emotional awareness. Hum. Brain Mapp. 34 (11), 2944-2958.

Dosenbach, N.U.F., Visscher, K.M., Palmer, E.D., Miezin, F.M., Wenger, K.K., Kang, H.C., Burgund, E.D., Grimes, A.L., Schlaggar, B.L., Petersen, S.E., 2006. A core system for the implementation of task sets. Neuron 50 (5), 799-812.

Nelson, S.M., Dosenbach, N.U.F., Cohen, A.L., Wheeler, M.E., Schlaggar, B.L., Petersen, S. E., 2010. Role of the anterior insula in task-level control and focal attention. Brain Struct. Funct. 214 (5-6), 669-680.

Milaneschi, Y., Lamers, F., Berk, M., Penninx, B.W.J.H., 2020. Depression Heterogeneity and Its Biological Underpinnings: Toward Immunometabolic Depression. Biol. Psychiatry 88 (5), 369-380.

Lamers, F., Milaneschi, Y., Vinkers, C.H., Schoevers, R.A., Giltay, E.J., Penninx, B.W.J.H., 2020. Depression profilers and immuno-metabolic dysregulation: longitudinal results from the NESDA study. Brain Behav. Immun. 88, 174-183. 